\title{
THERMAL DONOR GENERATION IN BORON- AND ALUMINIUM-DOPED CZOCHRALSKI SILICON
}

\author{
K. Kopalko, P. Kaczor, M. Godlewski \\ Institute of Physics, Polish Academy of Sciences \\ Al. Lotników 32/46, 02-668 Warszawa, Poland
}

AND T. GREgorkiewiCZ

Van der Waals-Zeeman Laboratorium, University of Amsterdam Valckenierstraat 65, NL-1018 XE Amsterdam, The Netherlands

\begin{abstract}
Generation of thermal donor centres in oxygen-rich silicon doped with boron and aluminium acceptors has been studied with the FTIR technique. It has been found that upon annealing $470^{\circ} \mathrm{C}$ two kinds of absorption series were generated. One of them belonged to the well-known first ionization level of silicon thermal (double) donors (TD's): $T D^{\circ} / T D^{+}$. The second series was identified with the so-called shallow thermal donors (STD's). The generation kinetics of the two series was followed for both kinds of acceptor doping and significant differences has been found. The results of the FTIR investigations were further compared with the magnetic resonance findings allowing for their mutual correlation and more general conclusions.
\end{abstract}

PACS numbers: 71.55.Ht, 78.50.Ge

\section{Introduction}

Thermal donors (TD's) in silicon are certainly among the most studied defect systems in solids. The interest stems here both from the applied point of view - oxygen-rich Czochralski-grown silicon accounts for more than $90 \%$ of substratesused in modern device manufacturing - as well as from the more fundamental approach, since TD's provide especially interesting case of aggregation phenomenon in solids. In spite of its long history - for more recent review see e.g. [1] - the issue of thermal donors is still not understood. Here the major existing controversy 


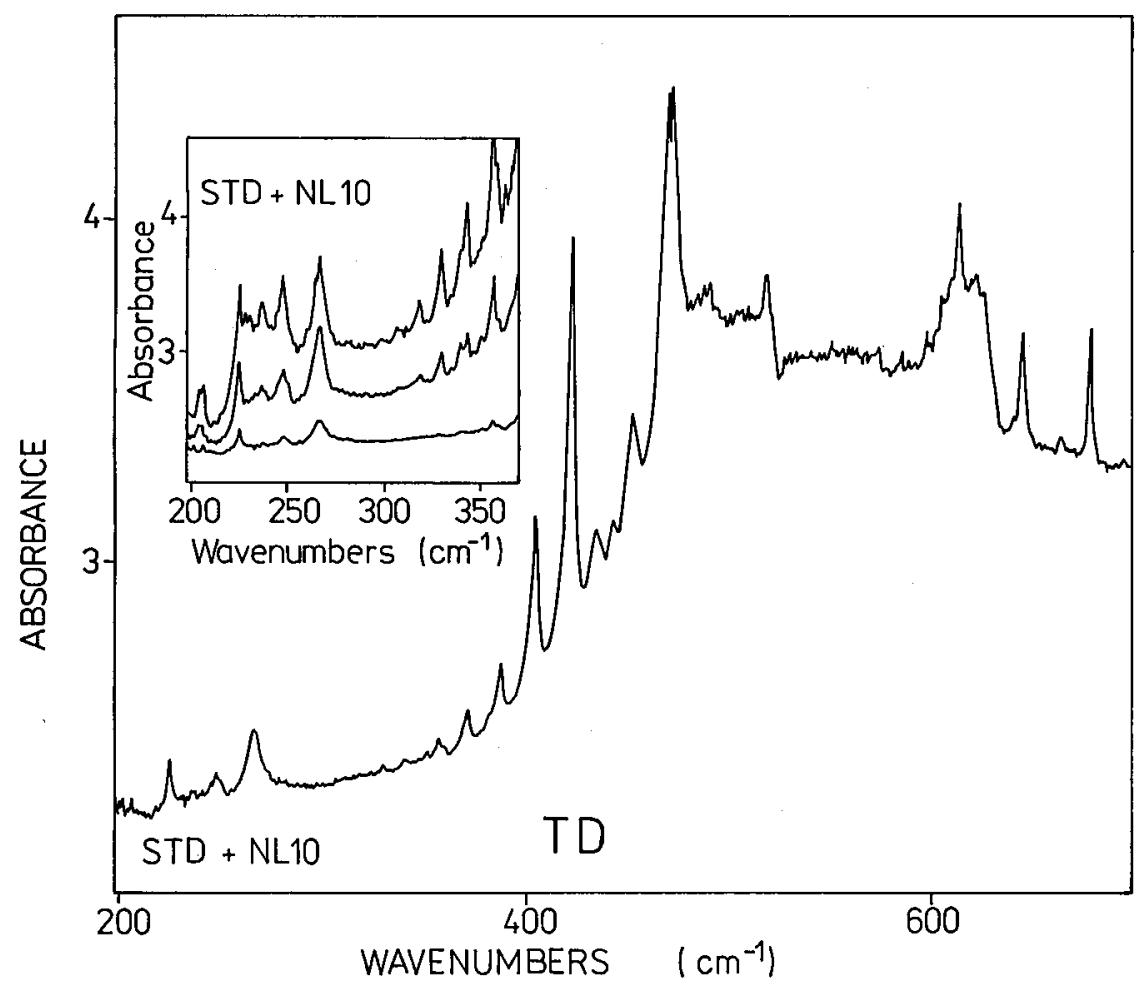

Fig. 1. Absorption spectrum of aluminium-doped Czochralski silicon sample (W38) annealed for $14 \mathrm{~h}$ at $470^{\circ} \mathrm{C}$. Individual absorption lines belonging to $T D^{\circ} / T D^{+}$and STD series are identified according to the literature.

follows from the fact that, while TD identification as oxygen clusters had positively been concluded, the diffusivity of oxygen in silicon is several orders of magnitude too low to account for very high concentration of TD centres. Consequently no definite undisputed microscopic model of TD structure exists at present.

In the past infrared absorption studies contributed significantly to our knowledge on TD's [2]. The detailed investigations revealed that two EMT-like ionization series could be related to TD's thus proving them to be (shallow) double donors with two energy levels $T D^{\circ} / T D^{+}$and $T D^{+} / T D^{++}$at $\approx 60 \mathrm{meV}$ and $\approx 150 \mathrm{meV}$, respectively. At the same time the multispecies character of TD has been discovered. Later photo-thermal ionization studies by Griffin et al. [3] revealed that yet another IR absorption series at $\approx 35 \mathrm{meV}$ labelled STD's (shallow thermal donors) could also be generated in oxygen-rich silicon by similar annealing procedure. The STD's were observed in very low concentration and after short annealing time of a few hours; longer heat treatment led to the anihilation of these centres.

At the same time in magnetic resonance studies [4] strong influence of the original acceptor doping on the generation kinetics of two TD-related Si-NL8 and 


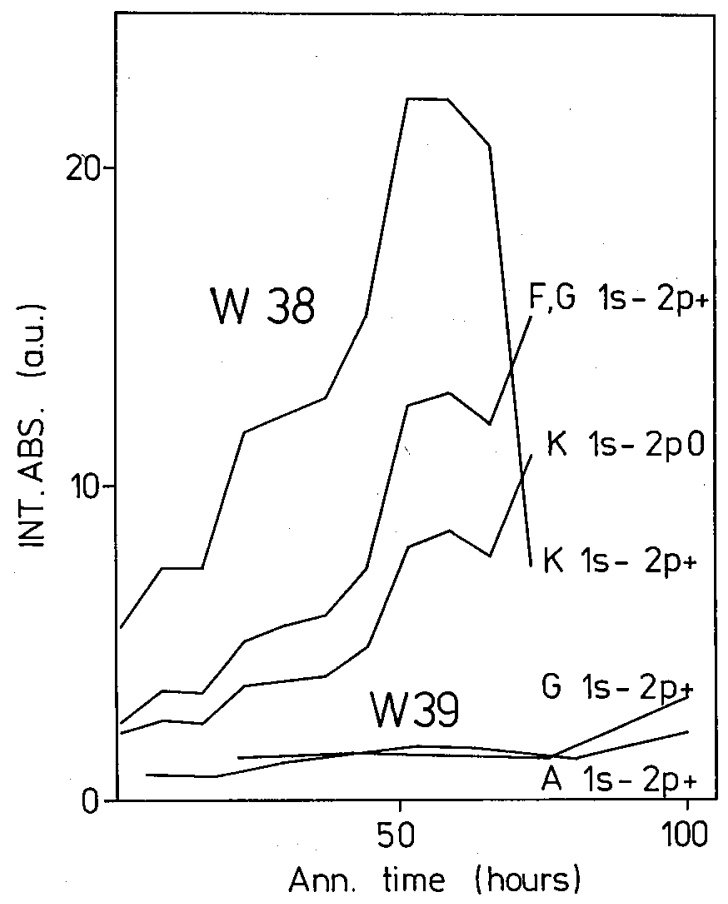

Fig. 2. Comparison of STD generation kinetics for aluminium-(W38) and boron-doped (W39) Czochralski silicon.

Si-NL10 paramagnetic centres has been concluded. Following this observation the current study focused on the acceptor influence as revealed by the infrared absorption studies.

\section{Experimental results and discussion}

Following the aim of the project two kinds of starting material have been used. The materials labelled W39 and W38 were high quality commercial Czochralski-grown low-carbon silicon (WASO grade) doped with boron $\left(1.3 \times 10^{16} \mathrm{~cm}^{-3}\right)$ and aluminium $\left(4 \times 10^{15} \mathrm{~cm}^{-3}\right)$, respectively. After the initial oxygen dispersion heat treatment the samples were subsequently annealed at $470^{\circ} \mathrm{C}$ for various time intervals up to 72 hours. The infrared absorption measurements were performed at liquid helium temperature with the Bomem FTIR spectrometer.

In both kinds of materials two thermal donor related series could be identified, namely the $T D^{\circ} / T D^{+}$and STD absorption lines. In Fig. 1 an examplary absorption spectrum as observed for W38 sample after relatively short annealing time of $14 \mathrm{~h}$ is depicted. Both series of lines are marked and identified according to the literature. The generation of the first TD ionization series was similar regardless the initial acceptor doping and in agreement with the expectations based on 
the previous results [2]. On the other hand the generation of STD series was surprizing because, according to the earlier findings [3], STD's should be observable only in highly resistive crystals and after very short annealing times. More recent studies related the STD-like lines either to the nitrogen doping [5] or uniquely to the high concentration of aluminium [6]. Our results clearly contradict all these findings showing that while the presence of aluminium dopant clearly seems to accelerate generation kinetics of STD's it is not absolutely necessary for their creation. Figure 2 presents the comparison of STD generation kinetics for two kinds of material used in this study; the strong enhancement for aluminium doping can clearly be concluded.

The current results seem to rule out the possibility of the STD series identification with either nitrogen-or aluminium-based oxygen single donor centre. At the same time the comparison of the generation kinetics as depicted in Fig. 2 with EPR data [4] provides strong evidence towards mutual identification of the STD series with Si-NL10 [7].

\section{References}

[1] L.C. Kimmerling, in Proceedings of the Symposium on Oxygen, Carbon, Hydrogen and Nitrogen in Crystalline Silicon, Materials Research Society Symposia Proceedings, Vol. 59, Eds. J.C. Mikkelsen Jr., S.J. Pearton, J.W. Corbett, S.J. Pennycook, MRS, Pittsburg 1986, p. 83.

[2] P. Wagner, J. Hage, Appl. Phys. A 49, 123 (1989).

[3] J.A. Griffin, H. Na varro, J. Weber, L. Genzel, J.T. Borenstein, J.W. Corbett, L.C. Snyder, J. Phys. C 19, L579 (1986).

[4] T. Gregorkiewicz, D.A. van Wezep, H.H.P.Th. Bekman, C.A.J. Ammerlaan, Phys. Rev. B 35, 3810 (1987).

[5] M. Suezawa, K. Sumino, H. Harada, T. Abe, Jpn. J. Appl. Phys. 25; L859 (1986).

[6] M. Claybourn, R.C. Newman, Mater. Sci. Forum 38-41, 613 (1989).

[7] T. Gregorkiewicz, H.H.P.Th. Bekman, C.A.J. Ammerlaan Phys. Rev. B 41, 12628 (1990). 